\title{
Analysis of Trends of 3 Major Benign Prostatic Hyperplasia Surgery in the Last 5 Years
}

\author{
Seung Ki Min, Byoung Hoon Kim, Kwi Bok Choi, In-Chang Cho
}

Department of Urology, National Police Hospital, Seoul, Korea

Purpose: The goal of this study is to analyze the trends in surgical management of Benign prostatic hyperplasia (BPH) in Korea during the last 5 years from 2014 to 2018.

Materials and Methods: We retrieved the medical statistics associated with the Healthcare Big Data Opening System available online. We analyzed the number of cases after 2014 for transurethral resection of prostate (TURP), photoselective vaporization of the prostate (PVP), and holmium laser enucleation of the prostate (HoLEP), respectively. We then analyzed and charted the cases according to the number of patients hospitalized or treated as outpatients, depending on age group, type of medical institutions, and the location of medical institutions. Results: The number of patients with BPH has increased steadily. The number of TURP and HoLEP procedures steadily increased, while the number of PVP interventions decreased dramatically. The number of HoLEP cases increased by $22 \%$ from 2014 to 2018 , which is the fastest rate among the three surgeries. In addition, the number of patients aged 75 years or older as well as the proportion of inpatient surgeries compared with outpatient treatments has increased. The number of cases undergoing TURP increased rapidly in general hospital and those treated with HoLEP increased in the general and tertiary referral hospitals.

Conclusions: Interventions using TURP and HoLEP has increased, and the age of patients undergoing surgery has increased gradually. Cases treated with TURP and HoLEP in general and tertiary referral hospitals showed an increasing trend from the metropolitan area to the province. (Korean J Urol Oncol 2019;17:160-167)

Key Words: Benign prostatic hyperplasia $\cdot$ Surgery $\cdot$ Korea $\cdot$ Trend

\section{INTRODUCTION}

Benign prostatic hyperplasia (BPH) is pathologically defined as hyperplasia of prostate epithelial cells and stromal cells. Clinically, BPH is traditionally considered as the progression of lower urinary tract symptoms (LUTS) charac-

Received October 2, 2019, Revised October 23, 2019,

Accepted October 25, 2019

Corresponding Author: In-Chang Cho

Department of Urology, National Police Hospital, 123 Songi-ro, Songpa-gu, Seoul 05715, Korea

E-mail: uroic@outlook.kr

Tel: +82-2-3400-1264, Fax: +82-2-431-3192

ORCID: https://orcid.org/0000-0001-8906-3478

- This study was supported by a Korean National Police Hospital Grant. terized by nocturia, weak urination stream, hesitancy, feeling of incomplete bladder emptying, urinary frequency, and urgency. Although the development of these LUTS is associated with other factors, such as changes in detrusor smooth muscle, the onset of these symptoms is usually due to bladder outlet obstruction caused by increased prostate size. ${ }^{1}$ The prevalence of annoying LUTS increases with age and is measured by the International Prostate Symptom Score, which is about $30 \%-40 \%$ in individuals aged over 50 years. $^{2}$ Due to the increase in the aging population in many developed and developing countries, the treatment of LUTS/BPH is an important health issue. According to the Foundation for Korean Urological Association, the number of patients who visited clinics in Korea due to BPH in 2010 was about 770,000 , with an annual average increase of

(i) (F) This is an Open Access article distributed under the terms of the Creative Commons Attribution Non-Commercial License (http://creativecommons.org/licenses/by-nc/4.0/) which permits unrestricted non-commercial use, distribution, and reproduction in any medium, provided the original work is properly cited. 2019 (C) Copyright The Korean Urological Oncology Society and The Korean Prostate Society. All Rights Reserved. 
$7.2 \%$, exceeding 1 million in $2014 .^{3}$

The primary treatment for $\mathrm{BPH}$ is medical therapy. However, surgical treatment is indicated for recurrent or unresponsive urinary retention, chronic urinary incontinence, recurrent urinary tract infection, bladder stones or bladder diverticulum, gross hematuria resistant to medication, and dilation of upper urinary tract due to BPH. In addition, despite conservative treatment or medication, surgery may be considered if there is insufficient improvement of urinary tract symptoms or postvoid residual urine. The type of surgery selected should be tailored to the patient considering various factors such as the severity of the LUTS, the effect on quality of life, the stability and cost of the surgical procedures, and complications. ${ }^{4,5}$

Surgical treatment for BPH is a radical intervention designed to eliminate prostate hypertrophy. The enlarged prostate adenoma can be surgically resected to eliminate or reduce the pressure in the urethra. Transurethral resection of the prostate (TURP) is a well-known and standard surgical treatment. With the development of instruments and techniques for TURP, the complication rate and mortality have been reduced. However, TURP was associated with a risk of complications such as hemorrhage, electrolyte abnormality, postoperative retrograde ejaculation, erectile dysfunction, bladder neck stenosis, urethral stricture, and reoperation. ${ }^{6}$ Advances in laser technology have been utilized in several medical fields and are also important in the treatment of BPH. However, early prostate laser surgeries such as transurethral ultrasound-guided laser-induced prostatectomy, which were introduced in the 1990s, improved symptoms in many patients, but the effects on increased urine flow rate and reduction of prostate size were insignificant and disappeared in the market. ${ }^{7}$ However, the newly developed lasers are not only effective in improving LUTS, increasing urine flow rate, and decreasing prostate size, but also show low rates of postoperative complication. According to the results of survey of the incidence of BPH surgeries conducted by the Foundation for Korean Urological Association, BPH surgery is most commonly utilized in the age group of 60-79 years, which shows the highest prevalence and accounts for $80 \%$ of all the cases of surgery conducted. ${ }^{8}$ In addition, the number of surgeries conducted in the age group of 80-89 years has increased recently. The objectives of this study were to analyze the number of cases for TURP, photoselective vaporization of the prostate (PVP), and holmium laser enucleation of the prostate (HoLEP) and to predict the demand and need for BPH surgery as an appropriate treatment and the associated social costs based on the trends in national aging.

\section{MATERIALS AND METHODS}

We analyzed the number of cases for TURP, PVP, and HoLEP based on the following parameters: hospitalization or outpatient treatment, age group, type and location of medical institution in Korea during the last 5 years from 2014 to 2018. First, we access the medical statistics available at http:/opendata.hira.or.kr/op/opc/olap3thDsInfo.do from the Healthcare Big Data Opening System provided by the Korea Health Insurance Review and Assessment Service. We used the code "N40" to identify the number of visits per year. Then, we clicked the 'inquiry' button on the upper right side of the web screen to check the number of patients who visited the medical institution for the disease code "N40" and the total amount of medical costs. Second, we accessed http://opendata.hira.or.kr/op/opc/olapDiagBhvInfo.do and analyzed the number of cases after 2014 by using R3975, R3976, and R3977 surgical codes for TURP, PVP, and HoLEP, respectively. We then analyzed and systemically charted the number of cases depending on hospital-

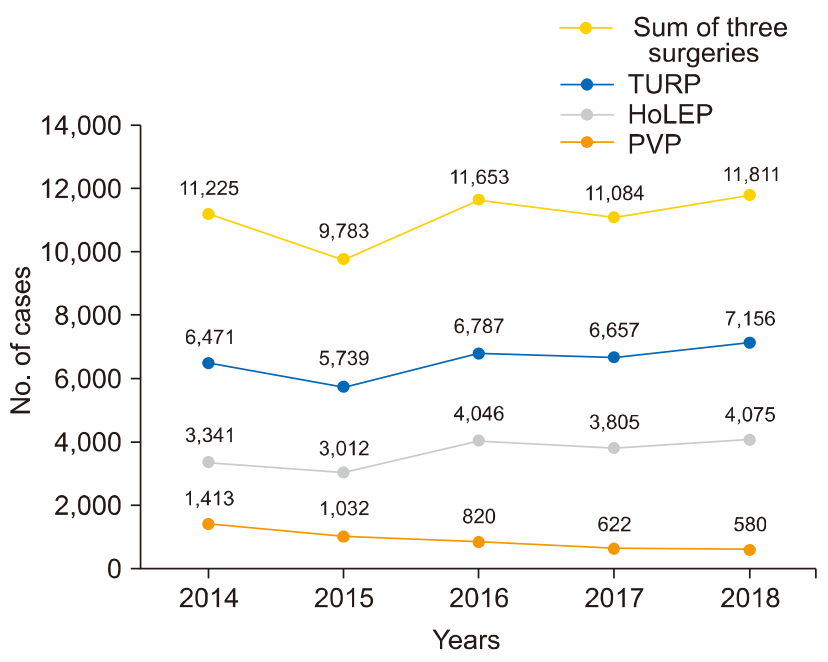

Fig. 1. Number of 3 major benign prostatic hyperplasia surgeries performed during last 5 years. TURP: transurethral resection of prostate, HoLEP: holmium laser enucleation of the prostate, PVP: photoselective vaporization of the prostate. 
ization or outpatient category, age group, and type and location of the medical institution each year. The study protocol was approved by the Institutional Review Board of our institution (No. 11100176-201909-HR-010). Informed consent requirements were waived because the study was based on open resources on the web.

\section{RESULTS}

After the number of patients who visited the clinic due to BPH (disease code N40) exceeded 1 million in 2014, the number increased steadily and exceeded 1.27 million in 2018. The total costs of medical care for BPH also increased, reaching about 195.9 billion won in 2018 from
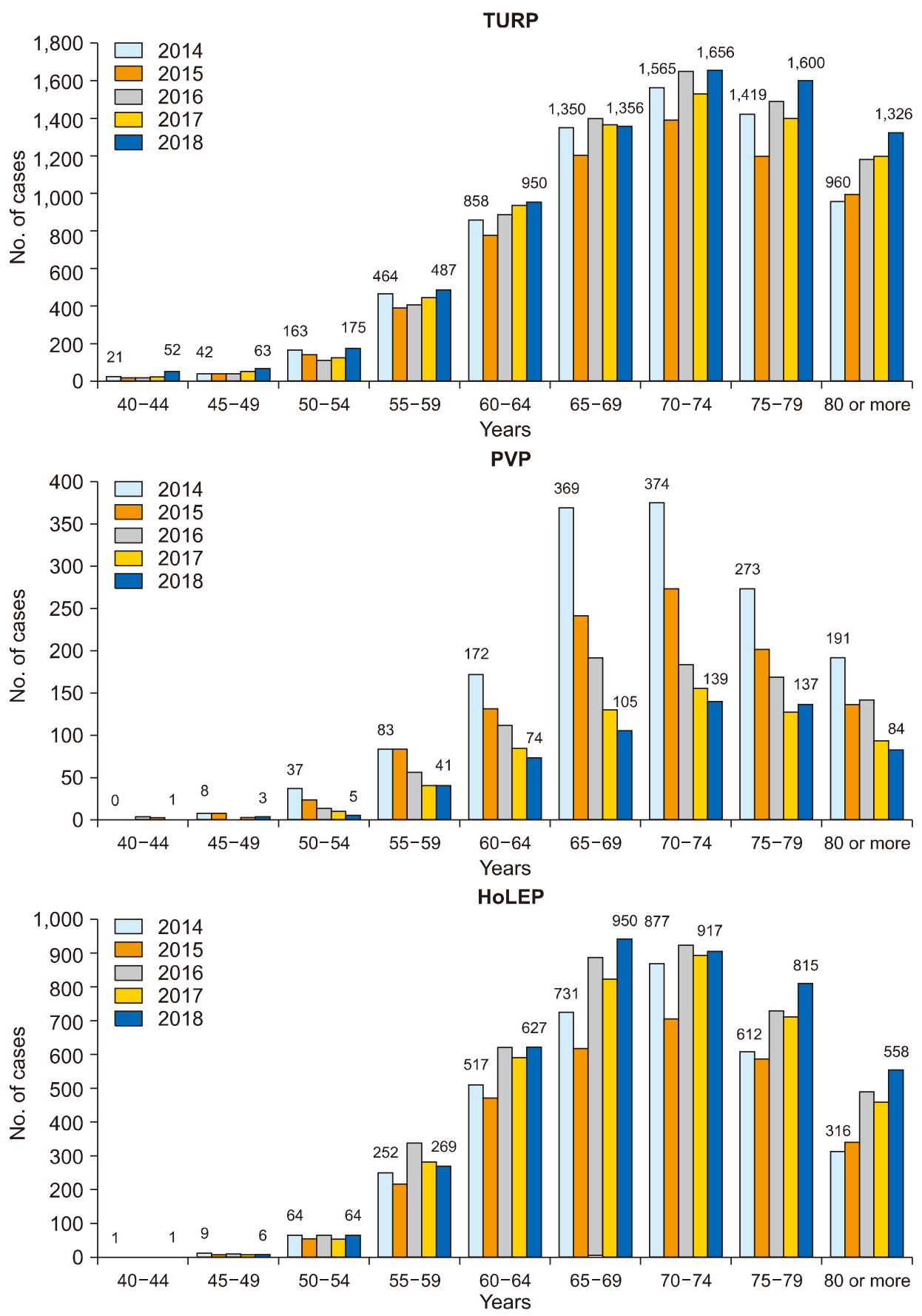

Fig. 2. Number of 3 major benign prostatic hyperplasia (BPH) surgeries performed during last 5 years according to age group. TURP: transurethral resection of prostate, PVP: photoselective vaporization of the prostate, HoLEP: holmium laser enucleation of the prostate. 
about 131.2 billion won in 2014 .

The total number of the 3 major surgeries for BPH decreased briefly from 11,225 cases in 2014 to 9,783 cases in 2015 , and increased by $5.2 \%$ to 11,811 cases in 2018 . When analyzed by each surgery, the TURP interventions increased by $10.6 \%$ from 6,471 cases in 2014 to 7,156 cases in 2018. PVP was reduced by $59 \%$ from 1,413 cases in 2014 to 580 cases in 2018 . HoLEP was increased by $22 \%$ from 3,341 cases in 2014 to 4,075 cases in 2018 (Fig. 1).

TURP and HoLEP were performed in $99 \%-100 \%$ of all hospitalized patients. PVP was performed in $13 \%$ of outpatient cases in 2014 , which declined to $4.4 \%$ of outpatient cases in 2018.

TURP was mostly used in patients aged 70-74 years (about 1,600 cases per year). However, the use of TURP was increased by $12.7 \%$ in the age group $75^{-79}$ years and $38.1 \%$ in patients above 80 years in 2018 compared with 2014. The use of PVP showed a declining trend in all age groups. HoLEP was most frequently performed in the age

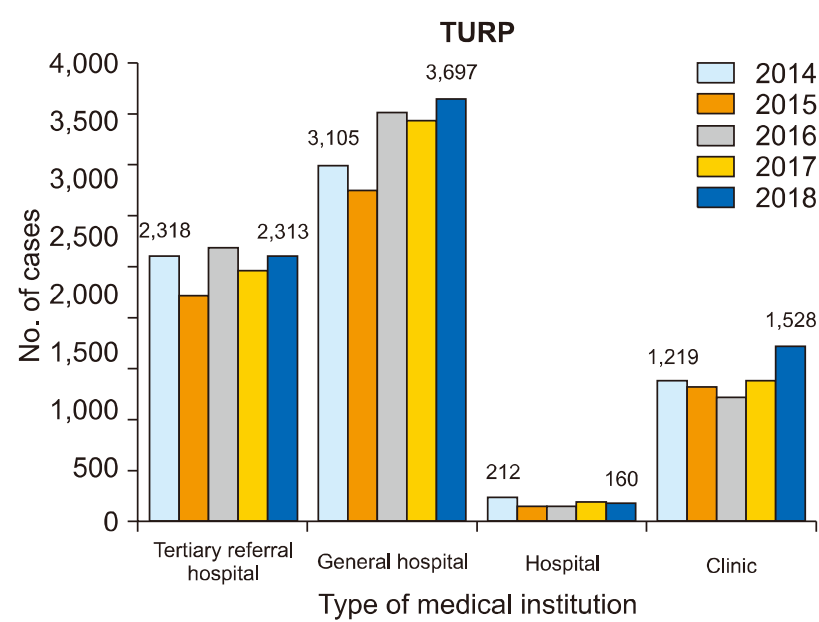

HoLEP

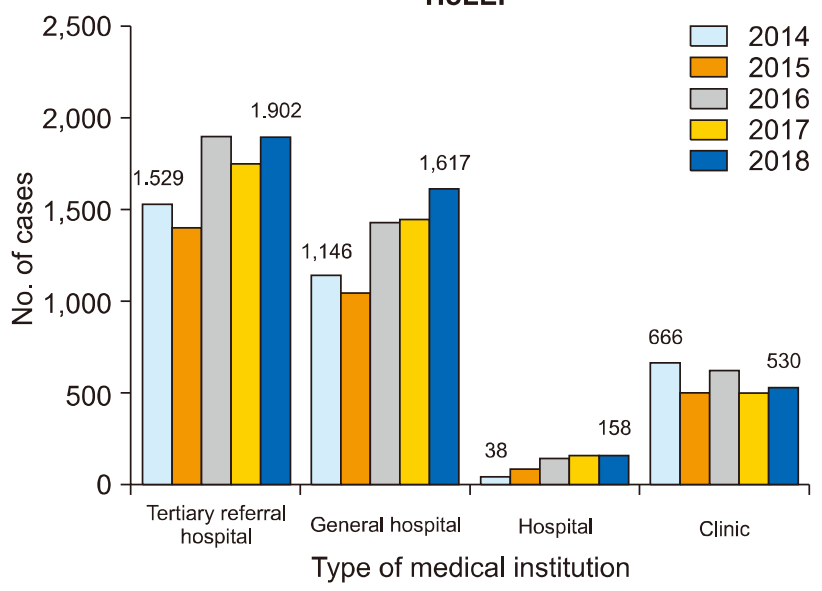

group of 70-74 years including nearly 900 cases per year in the past 5 years. During the year of 2018, HoLEP was performed more frequently in the 65-69 age group than in other age groups. Compared with 2014, in 2018, the number of cases increased by $33.2 \%$ in those aged $75-79$ years and by $77.6 \%$ in those 80 and older (Fig. 2).

Approximately 2,300 cases of TURP were performed annually in the tertiary referral hospitals. The number of cases in the general hospital increased by $19.1 \%$ in 2018 compared with 2014, and the number of TURPs was clinically prominent only in 2018. In tertiary referral hospitals, PVP was consistently performed in about 380 cases per year. In general hospitals, hospitals, and clinics, the cases of PVP decreased by more than $80 \%$ in 2018 compared with 2014 . The number of cases treated with HoLEP increased by $24.4 \%, 41.1 \%$ and $415.8 \%$ at the tertiary referral hospital, general hospital, and hospital, respectively, in 2018 compared with 2014. However, in clinics, the number of cases treated annually was similar during the study period (Fig. 3).

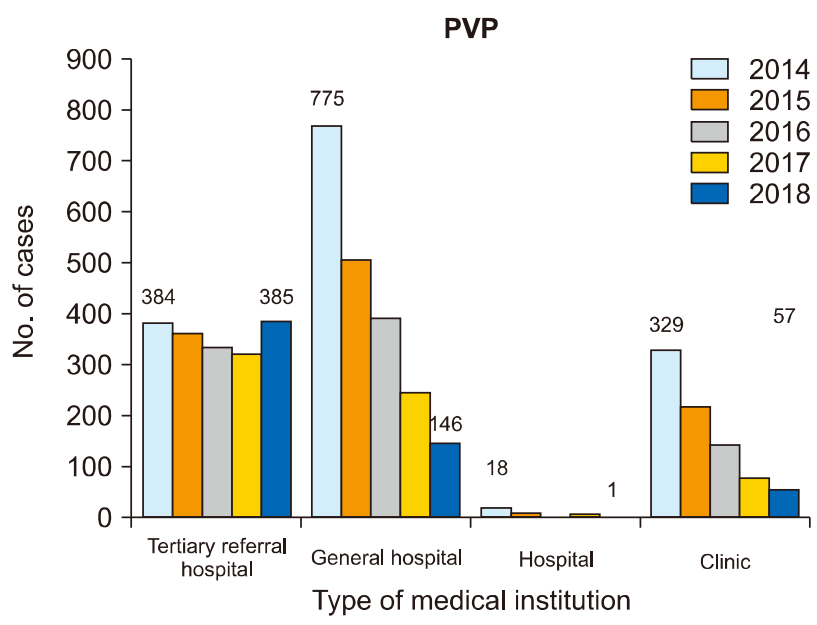

Fig. 3. Number of 3 major benign prostatic hyperplasia $(\mathrm{BPH})$ surgeries performed during last 5 years according to the type of medical institutions. TURP: transurethral resection of prostate, PVP: photoselective vaporization of the prostate, HoLEP: holmium laser enucleation of the prostate. 
In the analysis based on the location of medical institutions, TURP has become popular nationwide. Surprisin- gly in 2018, it surpassed in Incheon alone by $350 \%$ compared with 2014. PVP cases plummeted more than 50\% in
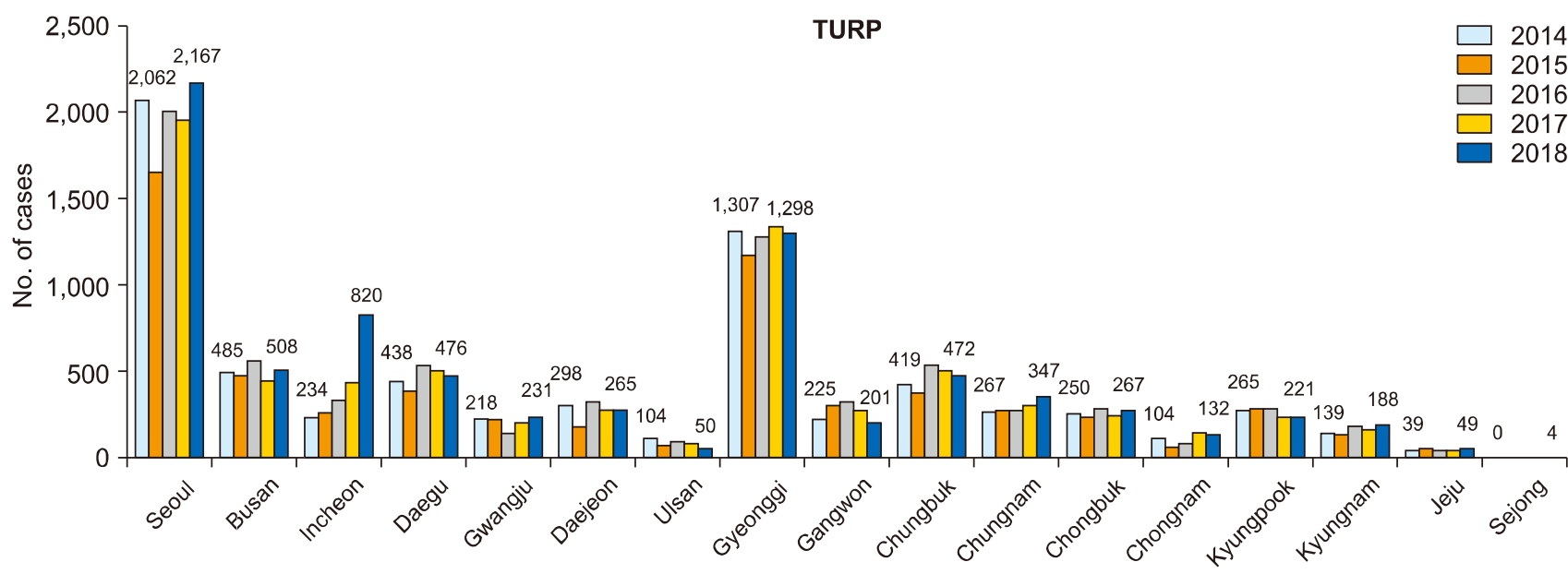

Location of medical institution
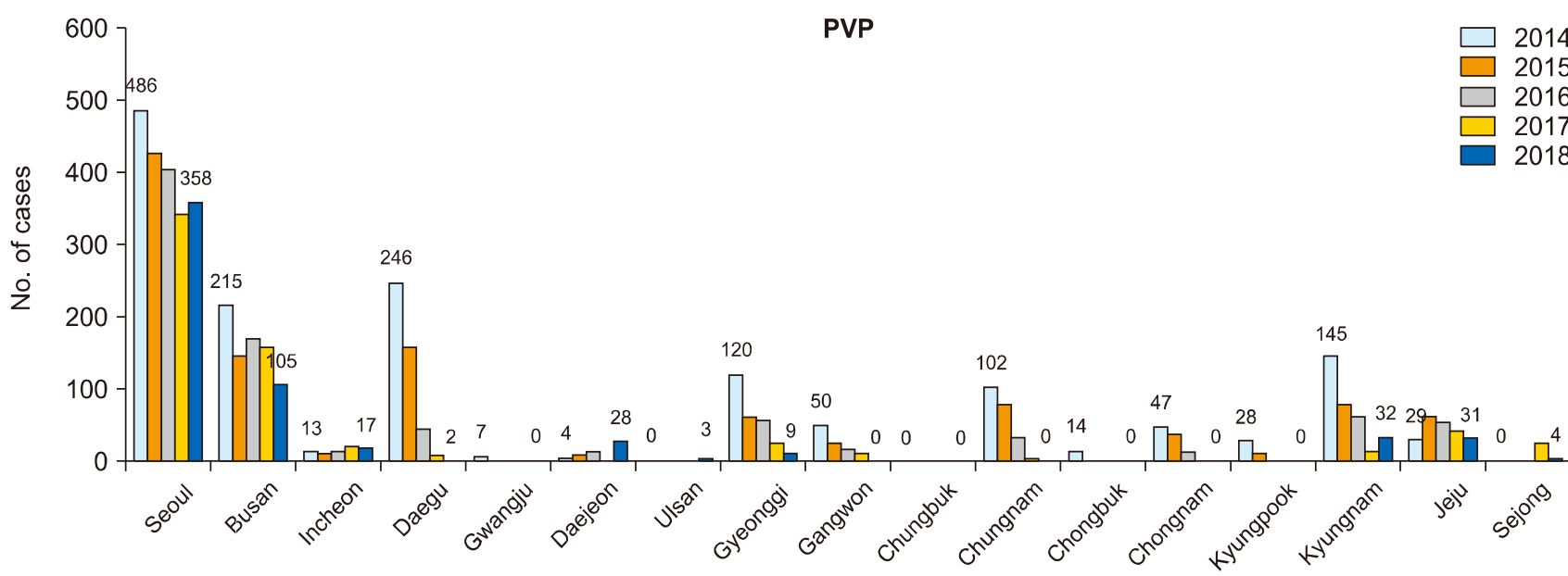

Location of medical institution

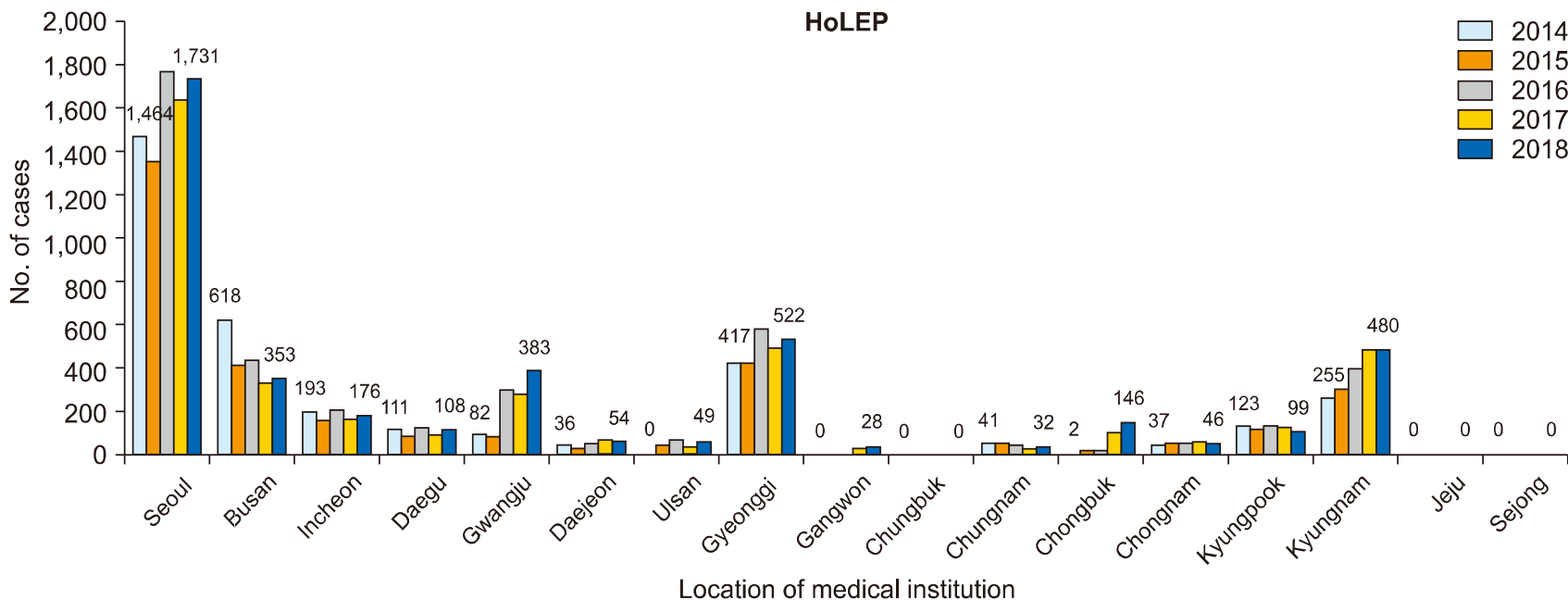

Fig. 4. Number of 3 major benign prostatic hyperplasia surgeries performed during last 5 years according to the location of medical institutions. TURP: transurethral resection of prostate, HoLEP: holmium laser enucleation of the prostate, PVP: photoselective vaporization of the prostate. 
2018 in all regions except for Seoul in 2014. HoLEP was implemented mainly in the metropolitan areas and big cities of 2014, but it spread throughout the country as the year progressed. In Seoul, Gwangju, Daejeon, Ulsan, Gyeonggi, Gangwon, Jeonbuk, Chonnam, and Gyeongnam, the number of cases increased. In Busan, Incheon, Chungnam, and Gyeongbuk, there was a slight decrease. In Chungbuk, Jeju, and Sejong, it was rarely performed until 2018 (Fig. 4).

\section{DISCUSSION}

$\mathrm{BPH}$ is the most common urological disease. Its surgical treatment has steadily progressed in the last 20 years. TURP has become a standard in BPH surgery for decades and has recently been challenged by various minimally invasive procedures using laser. In Korea, TURP is still the most commonly performed BPH surgery. In 2011, it accounted for less than $40 \%$ of all BPH surgeries in the United States. HoLEP is considered as an alternative endoscopic surgery for open prostatectomy. Several randomized trials of these 2 surgical methods showed almost similar results. HoLEP was superior to open surgery in the duration of catheterization, period of hospitalization, and blood loss. ${ }^{10}$ Stimulated by these merits, as the results of this study showed, the domestic epidemic of HoLEP is beginning in our country. However, in spite of its advantages in various prostate sizes, the use of HoLEP is limited by technical difficulties and steep learning curves. ${ }^{11}$ PVP refers to all kinds of prostate surgeries using a laser of 532-nm wavelength. Initially, the term was only used to refer to surgery using a potassium-titanyl-phosphate (KTP) laser, a crystal laser developed by Nd:YAG laser. However, it now includes surgery using lithium triborate laser, which improves the performance of KTP laser. The depth of tissue penetration by the KTP laser is $0.8 \mathrm{~mm}$ and is selectively absorbed by hemoglobin. Its energy is emitted in semicontinuous waves, and the removal of tissue is carried out by vaporization. ${ }^{12-14}$ Surface vaporization of the prostate generates intraprostatic cavity similar in shape to TURP. Because of its many advantages, such as minimal blood loss and short hospital stay, PVP has become popular and is rapidly spreading worldwide. Therefore, it was used as the primary surgical treatment for BPH. In the United States, PVP became popular, constituting about $30 \%$ of all BPH surgeries in 2008. The relatively short learning curve and fewer pre- and postoperative complications resulted in dramatic changes in surgical trend. ${ }^{2,9,15,16}$ However, it is associated with poor long-term results and high re-treatment rates in patients with large prostate size. ${ }^{17}$ This limitation is being overcome by performance improvement with the development of an XPS-180W GreenLight system that increases the output power and removes tissue more effectively. ${ }^{18-20}$

According to the results of this study, the number of patients with BPH (diagnostic code name N40) has increased steadily, with an annual rate of increase of about $6.2 \%$. The total cost of care has also increased, which is more than the increase in the number of patients, about $12.3 \%$ per year. However, the total number of 3 major surgeries for $\mathrm{BPH}$ increased relatively slowly, by $5.2 \%$ during recent 5 years. Various factors may contribute to this phenomenon. First, the inflation has led to escalating national insurance costs every year. Second, the number of HoLEP and TURP, which are relatively expensive operations, has increased steadily, while the number of PVP, a relatively inexpensive operation, declined sharply. In addition, the rate of reoperation for benign prostatic obstruction recurrence seemed slightly higher after PVP than after TURP. ${ }^{2}$ Third, the number of patients with poor general condition due to the increase in the number of older patients aged 75 or older is increasing the proportion of inpatient surgeries compared with outpatients.

In the future, the proportion of surgeries as well as medical treatment is expected to increase the management of BPH. In particular, the findings of this study showed that TURP is one of the BPH surgeries that is consistently favored by urologists, with an $10.6 \%$ increase from 2014 to 2018. The number of PVP interventions has declined sharply, and if this trend continues, its role in the treatment of $\mathrm{BPH}$ will be very limited. HoLEP procedures have increased by about $22 \%$ from 2014 to 2018, accounting for the fastest growth among the 3 major BPH surgeries. In the future, it is likely to become a routine surgery comparable to that of TURP. TURP and HoLEP have recorded rapid increases among patients aged over 75 years. In particular, the use of HoLEP was increased even in patients aged 6569 years with good general condition recently. However, the short- and long-term trends need to be analyzed. The use of 3 major BPH surgeries in tertiary referral hospitals was 
not increased significantly. However, the number of HoLEP procedures alone has increased in tertiary referral hospitals. In general hospitals, TURP and HoLEP cases showed a steady and rapid increase. The number of HoLEP procedures appears to be increasing at the general hospital level, but the number is still a small fraction of the total cases. In the past, PVP was preferred in many clinics, but its use has declined steeply. Recent trends suggest a preference for TURP to treat BPH. A similar number of HoLEP procedures appears to be conducted every year clinically. According to the location of medical institution, the rapid surge of TURP cases only in the Incheon area during 2018 is a very short-term finding. Therefore, it is necessary to observe future trends. HoLEP is a relatively new operation, with rapid clinical application across different provinces. It has been rarely implemented until 2018 in Chungbuk, Jeju, and Sejong. Thus, it is possible that there will be an increase in the number of cases treated with HoLEP in these areas. A number of factors may affect the frequency of HoLEP surgery in metropolitan and rural areas. However, the difference in accessibility to the technology due to the cost of equipment and apparatus is considered to be an important factor.

In Korea, national health insurance pays a large part of the costs related to national health care. When new treatments are developed, the cost burden is a very important issue in running the health insurance plans. Many studies have reported that HoLEP or GreenLight PVP is more cost-effective than TURP due to short hospital stays and fewer complications. ${ }^{21,22}$ Advances in BPH medication have led to a reduction in the rates of acute urinary retention and cases of BPH-related surgeries. ${ }^{23}$ However, patients who eventually require BPH surgery inevitably are elderly, with a large prostate gland, and manifest poor general condition, which inevitably increases the risk of surgery-related complications. ${ }^{24}$ In particular, the use of anticoagulants is inevitable in some older patients, in which case traditional surgeries such as TURP or laparotomy may be inappropriate. Based on the results of this study, the total number of BPH surgeries excluding PVP are increasing. Especially, the number of surgeries in elderly patients over 75 years has increased due to the rapid progression from the aging society to the aged society of Korea. Laser techniques with improved quality and performance and relatively less bleeding, fewer complications, and proven long-term results will be preferred by urologists.

\section{CONCLUSIONS}

In recent 5 years, the number of $\mathrm{BPH}$ surgeries in Korea has been steadily increasing. The number of cases treated with TURP and HoLEP has increased steadily, along with the surgical age. TURP at the general hospital level and HoLEP procedures at the tertiary referral hospital and the general hospital are being increasingly used from metropolitan to rural areas. In the future, the development of various lasers is expected to trigger the use of laser surgeries for $\mathrm{BPH}$.

\section{CONFLICT OF INTEREST}

The authors claim no conflicts of interest.

\section{REFERENCES}

1. Nickel JC, Méndez-Probst CE, Whelan TF, Paterson RF, Razvi H. 2010 Update: guidelines for the management of benign prostatic hyperplasia. Can Urol Assoc J 2010;4: 310-6.

2. Al-Ansari A, Younes N, Sampige VP, Al-Rumaihi K, Ghafouri A, Gul T, et al. GreenLight HPS 120-W laser vaporization versus transurethral resection of the prostate for treatment of benign prostatic hyperplasia: a randomized clinical trial with midterm follow-up. Eur Urol 2010;58: 349-55.

3. Health Insurance Review \& Assessment Service [Internet] Wonju (Korea): Health Insurance Review \& Assessment Service; c2015 [updated 2018 Sep 14; cited 2018 Sep 26]. Available from: http://opendata.hira.or.kr/op/opc/olapDiagBhvInfo.do.

4. Gratzke C, Bachmann A, Descazeaud A, Drake MJ, Madersbacher S, Mamoulakis C, et al. EAU Guidelines on the assessment of non-neurogenic male lower urinary tract symptoms including benign prostatic obstruction. Eur Urol 2015;67:1099-109.

5. Kaplan SA. Update on the american urological association guidelines for the treatment of benign prostatic hyperplasia. Rev Urol 2006;8 Suppl 4:S10-7.

6. Rassweiler J, Teber D, Kuntz R, Hofmann R. Complications of transurethral resection of the prostate (TURP)--incidence, management, and prevention. Eur Urol 2006;50: 969-79.

7. McCullough DL, Roth RA, Babayan RK, Gordon JO, 
Reese JH, Crawford ED, et al. Transurethral ultrasoundguided laser-induced prostatectomy: National Human Cooperative Study results. J Urol 1993;150(5 Pt 2):1607-11.

8. Medical observer [Internet] Seoul (Korea); c2015 [cited 2019 Apr 1]. Available from: http://www.monews.co.kr/news/art icleView.html?idxno $=88131$.

9. Malaeb BS, Yu X, McBean AM, Elliott SP. National trends in surgical therapy for benign prostatic hyperplasia in the United States (2000-2008). Urology 2012;79:1111-6.

10. Elzayat EA, Elhilali MM. Holmium laser enucleation of the prostate (HoLEP): long-term results, reoperation rate, and possible impact of the learning curve. Eur Urol 2007;52:1465-71.

11. Gilling PJ, Wilson LC, King CJ, Westenberg AM, Frampton CM, Fraundorfer MR. Long-term results of a randomized trial comparing holmium laser enucleation of the prostate and transurethral resection of the prostate: results at 7 years. BJU Int 2012;109:408-11.

12. Bach T, Muschter R, Sroka R, Gravas S, Skolarikos A, Herrmann TR, et al. Laser treatment of benign prostatic obstruction: basics and physical differences. Eur Urol 2012;61:317-25.

13. Gravas S, Bachmann A, Reich O, Roehrborn CG, Gilling PJ, De La Rosette J. Critical review of lasers in benign prostatic hyperplasia (BPH). BJU Int 2011;107:1030-43.

14. Malek RS, Kuntzman RS, Barrett DM. High power potassium-titanyl-phosphate laser vaporization prostatectomy. J Urol 2000;163:1730-3.

15. Capitán C, Blázquez C, Martin MD, Hernández V, de la Peña E, Llorente C. GreenLight HPS 120-W laser vaporization versus transurethral resection of the prostate for the treatment of lower urinary tract symptoms due to benign prostatic hyperplasia: a randomized clinical trial with 2-year follow-up. Eur Urol 2011;60:734-9.

16. Lukacs B, Loeffler $J$, Bruyère $F$, Blanchet $P$, Gelet $A$, Coloby $\mathrm{P}$, et al. Photoselective vaporization of the prostate with GreenLight 120-W laser compared with monopolar transurethral resection of the prostate: a multicenter randomized controlled trial. Eur Urol 2012;61:1165-73.

17. Hueber PA, Ben-Zvi T, Liberman D, Bhojani N, Gautam $\mathrm{G}$, Deklaj $\mathrm{T}$, et al. Mid term outcomes of initial 250 case experience with GreenLight 120W-HPS photoselective vaporization prostatectomy for benign prostatic hyperplasia: comparison of prostate volumes $<60 \mathrm{cc}, 60 \mathrm{cc}-100 \mathrm{cc}$ and $>100$ cc. Can J Urol 2012;19:6450-8.

18. Bachmann A, Muir GH, Collins EJ, Choi BB, Tabatabaei S, Reich OM, et al. 180-W XPS GreenLight laser therapy for benign prostate hyperplasia: early safety, efficacy, and perioperative outcome after 201 procedures. Eur Urol 2012;61:600-7.

19. Ben-Zvi T, Hueber PA, Liberman D, Valdivieso R, Zorn KC. GreenLight XPS $180 \mathrm{~W}$ vs HPS $120 \mathrm{~W}$ laser therapy for benign prostate hyperplasia: a prospective comparative analysis after 200 cases in a single-center study. Urology 2013;81:853-8.

20. Zorn KC, Liberman D. GreenLight $180 \mathrm{~W}$ XPS photovaporization of the prostate: how I do it. Can J Urol 2011; 18:5918-26.

21. Fraundorfer MR, Gilling PJ, Kennett KM, Dunton NG. Holmium laser resection of the prostate is more cost effective than transurethral resection of the prostate: results of a randomized prospective study. Urology 2001;57:454-8.

22. Liatsikos E, Kyriazis I, Kallidonis P, Sakellaropoulos G, Maniadakis N. Photoselective GreenLight ${ }^{\mathrm{TM}}$ laser vaporization versus transurethral resection of the prostate in Greece: a comparative cost analysis. J Endourol 2012;26: 168-73.

23. McConnell JD, Roehrborn CG, Bautista OM, Andriole GL Jr, Dixon CM, Kusek JW, et al. The long-term effect of doxazosin, finasteride, and combination therapy on the clinical progression of benign prostatic hyperplasia. $\mathrm{N}$ Engl J Med 2003;349:2387-98.

24. Choi SY, Kim TH, Myung SC, Moon YT, Kim KD, Kim $\mathrm{YS}$, et al. Impact of changing trends in medical therapy on surgery for benign prostatic hyperplasia over two decades. Korean J Urol 2012;53:23-8. 